\title{
PARTITIONING A SET
}

\author{
R. H. BING
}

1. Introduction. Suppose space has a metric $D(x, y)$.

Under certain conditions in calculus we define $\int_{b}^{a} f(x) d x$ to be equal to $\lim \sum f\left(\xi_{i}\right) \Delta x_{i}$. The dividing of the interval from $a$ to $b$ into a finite number of pieces with lengths $\Delta x_{i}$ is a partitioning of the interval from $a$ to $b$. Similarly, partitioning may be used in integrating over an arbitrary set. The partitioning may provide a basis for a measure on the range of the function which is being integrated. We shall define partitioning on sets for which there is no accepted unit of measure such as length, area, or volume.

The notion of partitioning may be used to assign a convex metric to certain sets. See Theorems $7,8,9$, and 10 . Theorem 8 provides an answer to a question raised by Menger in [3]. ${ }^{1}$ See [1] for a discussion of and references to partial solutions of this problem. Further applications of partitioning are treated in my paper Complements of continuous curves, appearing in Fund. Math.

Most of the results of this paper (including Theorem 7 but not Theorems 1 and 8) were contained in an address I gave before the Mathematics Club at the University of Chicago in May, 1948. After finishing Theorems 1 and 8 in August, I found that Moise, working independently, had obtained a solution [4] to the above mentioned problem by Menger six weeks earlier.

Partitioning. A set $M$ can be partitioned if for each positive number $\epsilon$ there is a finite collection $G$ of connected mutually exclusive open subsets of $M$ such that each element of $G$ is of diameter less than $\epsilon$ and the sum of the elements of $G$ is dense in $M$. We shall say that $G$ is an $\epsilon$-partitioning of $M$.

Refinement. If $G$ and $H$ are two partitionings of $M$, we say that $G$ is a refinement of $H$ if each element of $G$ is a subset of an element of $H$.

Property S. A set $M$ has property $\mathrm{S}$ if for each positive number $\epsilon, M$ is the sum of a finite number of connected subsets such that the diameter of each of these subsets is less than $\epsilon$. This property is discussed in $[5,6,7,8]$.

Convex metric. A metric $E$ for a set $M$ is convex if for each pair of points $x, y$ of $M$ there is a point $z$ of $M$ such that $E(x, z)=E(y, z)$

Presented to the Society, September 10,1948; received by the editors September $2,1948$.

${ }^{1}$ Numbers in brackets refer to the bibliography at the end of the paper. 
$=E(x, y) / 2$. The metric $F$ for $M$ is almost convex [2] if for each pair of points $x, y$ of $M$ and each positive number $\epsilon$ there is a point $z$ of $M$ such that

$$
|F(x, z)-F(x, y) / 2|+|F(y, z)-F(x, y) / 2|<\epsilon .
$$

2. Partitionable sets. Theorem 1 characterizes sets that can be partitioned. First we consider two lemmas that will be used.

Lemma 1. For each connected set $M$ with property $S$ there is a compact locally connected continuum $H$ and a homeomorphism $T$ of $M$ into a dense subset $H^{\prime}$ of $H$ such that the diameter of each connected subset $X$ of $M$ is the same as the diameter of $T(X)$ and for each connected open subset $R$ of $H, T^{-1}\left(R \cdot H^{\prime}\right)$ is connected.

Proof. The continuum $H$ is the complete enclosure of the relative distance space for $M$. See [8; pp. 154-158].

Lemma 2. If $M$ is a connected set with property $\mathrm{S}$ and $H$ and $K$ are subsets of $M$ at a positive distance from each other, then there is a finite collection of connected, mutually exclusive open subsets of $M$ such that the sum of this collection is dense in $M$, no element of this collection intersects both $H$ and $K$, and each element of this collection that intersects $K$ has property $\mathrm{S}$.

Proof. We shall prove the lemma for the case where $M$ is closed and compact. It will follow from Lemma 1 for the general case.

Let $H^{\prime}$ and $K^{\prime}$ be two sets at a positive distance from each other, containing $H$ and $K$ respectively, such that each point of $M$ belongs to an arc of diameter less than $1 / 2$ that intersects $H^{\prime}+K^{\prime}$. Denote by $H_{1}$ (or $K_{1}$ ) the set of all points of $M$ that belong to a connected subset of $M$ which intersects $H^{\prime}$ (or $K^{\prime}$ ) and is of diameter less than $D\left(H^{\prime}, K^{\prime}\right) / 3$. We note that $H_{1}$ and $K_{1}$ have only a finite number of components.

Let $W$ be a finite collection of points of $M$ such that each point of $M$ belongs to an arc in $M$ of diameter less than $1 / 4$ that intersects $W$. There is a finite collection $A$ of arcs such that each element of $A$ lies in $M-\bar{K}_{1}$ and intersects $H_{1}$ while $A^{*}$, the sum of the elements of $A$, contains each point of $W$ which belongs to a component of $M-\bar{K}_{1}$ which intersects $H_{1}$. Let $B$ be a finite collection of arcs such that $A^{*}+B^{*}$ contains $W$ and each element of $B$ is of diameter less than $1 / 2$, lies in $M-\left(\bar{H}_{1}+A^{*}\right)$, and intersects $K_{1}$.

Denote by $\mathrm{H}_{2}$ (or $K_{2}$ ) the set of all points that belong to a connected subset of $M$ which intersects $H_{1}+A^{*}$ (or $K_{1}+B^{*}$ ) and is of diameter less than $D\left(H_{1}+A^{*}, K_{1}+B^{*}\right) / 3$. We note that each point 
of $K_{2}$ belongs to a connected subset of $K_{2}$ which intersects $K_{1}$ and is of diameter less than $1 / 2+1 / 6$.

Similarly, there exist open subsets $H_{3}, K_{3}, H_{4}, K_{4}, \cdots$ of $M$ such that (1) $H_{i}$ is at a positive distance from $K_{i}$, (2) $H_{i+1}$ and $K_{i+1}$ contain $\bar{H}_{i}$ and $\bar{K}_{i}$ respectively, (3) each component of $H_{i+1}$ contains a component of $H_{i}$, (4) each point of $K_{i+1}$ belongs to a connected subset of $K_{i+1}$ that intersects $K_{i}$ and is of diameter less than $1 / 2^{i}+1 /\left(3 \cdot 2^{i}\right)$, and (5) each point of $M$ belongs to an arc of diameter less than $1 / 2^{i}$ that intersects $H_{i}+K_{i}$. Now $\sum\left(H_{i}+K_{i}\right)$ is dense in $M$ and has only a finite number of components while $\sum K_{i}$ has property $\mathrm{S}$.

Theorem 1. A necessary and sufficient condition that a set $M$ can be partitioned is that it have property $\mathrm{S}$.

Proof of sufficiency. We show that $M$ can be partitioned by showing that each of its components $C$ can be partitioned.

Let $\left(p_{1}, p_{2}, \cdots, p_{n}\right)$ be a finite collection of points of $C$ such that each point of $M$ is at a distance from $\sum p_{i}$ of less than $\epsilon / 4$. Let $H_{i}$ be the set of all points of $C$ that are at a distance from $p_{i}$ of less than $\epsilon / 4$ and $K_{i}$ be the set of those that are at a distance from $p_{i}$ of more than $\epsilon / 2$.

By Lemma 2 there are two mutually exclusive open subsets $U_{1}$ and $V_{1}$ of $C$ such that $U_{1}+V_{1}$ is dense in $C, U_{1}$ has a finite number of components and contains $H_{1}$, and $V_{1}$ has property $\mathrm{S}$ and contains $K_{1}$. Also, there are two open subsets $U_{2}$ and $V_{2}$ of $V_{1}$ such that $U_{2}+V_{2}$ is dense in $V_{1}, U_{2}$ contains $V_{1} \cdot H_{2}$ and has a finite number of components, while $V_{2}$ contains $V_{1} \cdot K_{2}$ and has property $\mathrm{S}$. Similarly, we define sets $U_{3}, V_{3}, U_{4}, V_{4}, \cdots, U_{n-1}, V_{n-1}$. The components of $U_{1}+U_{2}+\cdots+U_{n-1}+V_{n-1}$ are finite in number, each is of diameter less than $\epsilon$, and their sum is dense in $M$.

3. Refinements of partitionings. We shall be interested in partitioning a set in such a way that each of the pieces into which it is partitioned can be partitioned. These pieces must have property $S$ if this is to be done.

THEOREM 2. If $H$ and $K$ are two connected subsets of the connected partitionable set $M$ and $\epsilon$ is a positive number such that any connected subset of $M$ intersecting both $H$ and $K$ is of diameter more than $\epsilon$, then there are two connected, mutually exclusive open subsets $U$ and $V$ of $M$ containing $H$ and $K$ respectively such that $U+V$ has property $\mathrm{S}$ and is dense in $M$.

Proof. The general case will follow from Lemma 1 if we prove the 
case where $M$ is a compact continuum. Hence, we suppose it is one.

For each integer $i$, let $G_{i}$ be a $1 / i$-partitioning of $M$. Let $H^{\prime}$ and $K^{\prime}$ be mutually exclusive subcontinua of $M$ containing $H$ and $K$ respectively such that each element of $G_{2}$ intersects $H^{\prime}+K^{\prime}$. Let $U_{1}$ (or $V_{1}$ ) be the set of all points that belong to a connected subset of $M$ of diameter less than $D\left(H^{\prime}, K^{\prime}\right) / 3$ that intersects $H^{\prime}$ (or $K^{\prime}$ ).

Let $A$ and $B$ be finite collections of arcs such that (1) each element of $A$ intersects $U_{1}$ while each element of $B$ intersects $V_{1}$, (2) each element of $A+B$ is a subset of an element of $G_{2},(3)$ no element of $A$ intersects either $\bar{V}_{1}$ or an element of $B$, while no element of $B$ intersects $\bar{U}_{1}$, and (4) each element of $G_{4}$ contains a point of $A^{*}+B^{*}$.

Let $U_{2}$ (or $V_{2}$ ) be the set of all points that belong to a connected subset of $M$ of diameter less than $D\left(U_{1}+A^{*}, V_{1}+B^{*}\right) / 3$ that intersects $U_{1}$ (or $V_{1}$ ). We note that each point of $U_{2}$ (or $V_{2}$ ) belongs to a connected subset of $U_{2}$ (or $V_{2}$ ) of diameter less than $1 / 2+D\left(U_{1}+A^{*}\right.$, $\left.V_{1}+B^{*}\right) / 3<1 / 2+1 / 6$ that intersects $U_{1}$ (or $V_{1}$ ).

Similarly, we obtain connected open subsets $U_{3}, V_{3}, U_{4}, V_{4}, \cdots$ such that (1) $U_{i+1}$ contains $\bar{U}_{i}$ while $V_{i+1}$ contains $\bar{V}_{i}$, (2) $U_{i}+V_{i}$ intersects each element of $G_{2^{i}}$, and (3) each point of $U_{i+1}$ (or $V_{i+1}$ ) belongs to a connected subset of $U_{i+1}$ (or $V_{i+1}$ ) which is of diameter less than $1 / 2^{i}+1 /\left(3 \cdot 2^{i}\right.$ ) and intersects $U_{i}$ (or $V_{i}$ ). Then $U=\sum U_{i}$ and $V=\sum V_{i}$ are the required sets.

Each of the following three theorems follows from repeated applications of the theorem preceding it.

TheOREM 3. If $H$ is a finite collection of connected subsets of the connected partitionable set $M$ and $\epsilon$ is a positive number such that no connected subset of $M$ of diameter less than $\epsilon$ intersects two elements of $H$, then there is a finite collection $U$ of connected, mutually exclusive open subsets of $M$ such that each element of $U$ has property $\mathrm{S}$ and contains one and only one element of $H$, each element of $H$ is contained in an element of $U$, and the sum of the elements of $U$ is dense in $M$.

THEOREM 4. If $M$ is partitionable, then for each positive number $\epsilon$ there is an $\epsilon$-partitioning $G$ of $M$ such that each element of $G$ has property $\mathrm{S}$.

THEOREM 5. If $M$ is a partitionable set, there is a sequence $G_{1}, G_{2}, \ldots$ such that $G_{i}$ is a $1 / i$-partitioning of $M$ and $G_{i+1}$ is a refinement of $G_{i}$.

Theorem 6. Suppose space has a complete metric and $R$ is a connected domain with property $\mathrm{S}$ and a nondegenerate boundary $B$. Then $R$ contains a connected set $E$ with property $S$ such that $\bar{E}=E+B$, but no connected subset of $E$ has this property. Also, $E$ is topologically 
equivalent to a subset of a dendron, and any nondegenerate continuum in $E$ is a dendron with a finite number of ends.

Proof. Let $G_{1}, G_{2}, \cdots$ be a sequence such that $G_{i}$ is a $1 / i$-partitioning of $R$ and $G_{i+1}$ is a refinement of $G_{i}$. Denote the collection of closures of elements of $G_{i}$ by $H_{i}$. Let $H_{1}^{\prime}$ be a subset of $H_{1}$ irreducible with respect to covering $B$ and $H_{i+1}^{\prime}$ be a subcollection of $H_{i+1}$ such that each element of $H_{i+1}^{\prime}$ is a subset of an element of $H_{i}^{\prime}$, and $H_{i+1}^{\prime}$ is irreducible with respect to covering $B$.

Let $m_{1}$ be an integer so large that $H_{m_{1}}^{\prime}$ has more than one element. There is a dendron $T_{1}$ in $R$ which intersects each element of $H_{m_{1}}^{\prime}$ but which contains no subcontinuum doing so. Let $m_{2}$ be an integer so large that $D\left(T_{1}, B\right)>3 / m_{2}$. There is a dendron $T_{2}$ such that (1) $T_{2}$ contains $T_{1}$, (2) $T_{2}$ intersects each element of $H_{m_{2}}^{\prime}$, (3) each component of $T_{2}-T_{1}$ is a subset of an element of $G_{m_{1}}$, and (4) no subcontinuum of $T_{2}$ has these properties. Similarly, we define $T_{3}, T_{4}, \cdots$. Then $E=\sum T_{i}$.

4. Convexification of partitionable sets. We can assign a convex metric to certain partitionable sets.

THEOREM 7. If $M$ is a compact partitionable continuum, it can be assigned a convex metric.

First Proof of Theorem 7. We shall define a sequence $E_{1}(x, y)$, $E_{2}(x, y), \cdots$ of functions on the pairs of points of $M$ such that $E(x, y)=\lim E_{i}(x, y)$ is a convex metric for $M$.

Let $G_{1}, G_{2}, \cdots$ be a sequence such that $G_{i}$ is a $1 / i$-partitioning of $M$ and $G_{i+1}$ is a refinement of $G_{i}$. There is a dendron $T_{1}$ in $M$ such that $T_{1}$ intersects each element of $G_{1}$, and there is a convex metric $F_{1}$ for $T_{1}$ such that the diameter of $T_{1}$ under $F_{1}$ is less than $1 / 4$. Let $U_{1}$ be the set of all elements $u$ such that $u$ is a component of $g-g \cdot T_{1}$ for some element $g$ of $G_{1}$. Denote by $V_{1}$ the set of all continua $v$ such that $v$ is either a point of $T_{1}$ or the closure of an element of $U_{1}$.

If $v$ and $w$ are two elements of $V_{1}$, we define $E_{1}(v, w)$ to be the greatest lower bound of all numbers of the sort $r / 2+s$, where some continuum is the sum of $v, w, r$ other elements of $V_{1}$ and some arcs in $T_{1}$ whose total length under $F_{1}$ is $s$.

Let $m_{2}$ be an integer so large that $D(v, w)>3 / m_{2}$ if $v$ and $w$ are elements of $V_{1}$ such that $E_{1}(v, w)>1 / 16$. In each element $u_{i}$ of $U_{1}$ that contains an element of $G_{m}$ there is a dendron $T_{2, i}$ which intersects each element of $G_{m g}$ that intersects $u_{i}$. It may be noted that there are only a finite number of such $u_{i}$ 's. Let $F_{2, i}$ be a convex metric for $T_{2, i}$ such that the diameter of $T_{2, i}$ under $F_{2, i}$ is less than $1 / 16$. 
Let $U_{2}$ be the collection of all components of the sort $g-g$ - $\left(T_{1}+\sum T_{2, i}\right)$, where $g$ denotes an element of $G_{m_{2}}$. Let $V_{2}$ designate the set of all continua $v$ such that $v$ is either a point of $T_{1}+\sum T_{2, i}$ or the closure of an element of $U_{2}$.

If $v$ and $w$ are two elements of $V_{2}$, we define $E_{2}(v, w)$ to be the greatest lower bound of all numbers of the type $r / 4+s$, where some continuum is the sum of $v, w, r$ other elements of $V_{2}$ and a collection of $\operatorname{arcs}$ in $T_{1}+\sum T_{2, i}$ whose total length under their respective $F$ 's is $s$.

Similarly, we define $m_{3}, G_{m_{3}}, T_{3, j}, F_{3, j}, U_{3}, V_{3}, E_{3}(v, w), m_{4}, G_{m_{4}}$, $T_{4, j}, F_{4, j}, U_{4}, V_{4}, E_{4}(v, w), \cdots$. In general, $m_{i+1}$ is an integer so large that

(1) $\quad D(v, w)>3 / m_{i+1} \quad$ if $\quad E_{i}(v, w)>1 / 4^{i+1} \quad\left(v, w\right.$ elements of $\left.V_{i}\right)$.

Consider an element $u_{i, n}$ of $U_{i}$ that contains an element of $G_{m_{i+1}}$. Let $K_{i, n}$ denote the collection of all continua $k$ such that $k$ is the closure of a component of the common part of $u_{i, n}$ and an element of $G_{m_{i+1}}$. Denote by $K_{i, n}^{\prime}$ the finite collection of all elements $k$ of $K_{i, n}$ such that $k$ intersects an element of $V_{i}$ which does not intersect any component of $T_{1}+\sum T_{2, j}+\cdots+\sum T_{i, j}$ which intersects $k$. There is a dendron $T_{i+1, n}$ in $u_{i, n}$ such that $T_{i+1, n}$ intersects each element of $K_{i, n}^{\prime}$ and each element of $G_{m_{i+1}}$ which intersects $u_{i, n}$. Let $F_{i+1, n}$ be a convex metric for $T_{i+1, n}$ such that

$$
\text { diameter } T_{i+1, n} \text { under } F_{i+1, n}<1 / 4^{i+1} \text {. }
$$

Denote by $U_{i+1}$ the collection of all components of sets of the sort $g-g \cdot\left(T_{1}+\sum T_{2, j}+\cdots+\sum T_{i+1, j}\right)$, where $g$ denotes an arbitrary element of $G_{m_{i+1}}$. The elements of $V_{i+1}$ are the points of $T_{1}+\sum T_{2, j}$ $+\cdots+\sum T_{i+1, j}$ and the closures of the elements of $U_{i+1}$.

Let

$E_{i+1}(v, w)=$ greatest lower bound $\left(r / 2^{i+1}+s\right) \quad\left(v, w\right.$ elements of $\left.V_{i+1}\right)$, where some continuum is the sum of $v, w, r$ other elements of $V_{i+1}$ and a collection of arcs in $T_{1}+\sum T_{2, j}+\cdots+\sum T_{i+1, j}$ whose lengths under their respective $F$ 's total $s$. It may be noted that if $v$ intersects $w, E_{i}(v, w)=0$.

If $p$ and $q$ are two points of $M$, we define $E_{i}(p, q)$ to be the greatest lower bound of all numbers $E_{i}(v, w)$, where $v$ and $w$ are elements of $V_{i}$ containing $p$ and $q$ respectively. We shall show that $\lim E_{i}(p, q)$ $=E(p, q)$ exists and $E(x, y)$ is a convex metric for $M$.

Using (1) we find that if some continuum is the sum of the elements $v$ and $w$ of $V_{i}$ and $n$ elements of $V_{i+1}$, then $E_{i}(v, w)<n / 2^{i+1}$. From this 
and the fact that two elements of $V_{i+1}$ are subsets of the same element of $V_{i}$ if they intersect the same arc in $\sum T_{i+1, j}$, we find that if $v$ and $w$ are two elements of $V_{i+1}$, then there are elements $v^{\prime}$ and $w^{\prime}$ of $V_{i}$ containing $v$ and $w$ respectively such that $E_{i}\left(v^{\prime}, w^{\prime}\right) \leqq E_{i+1}(v, w)$. Hence, $E_{i}(p, q)$ is a monotone nondecreasing function of $i$.

If a component $C$ of $T_{1}+\sum T_{2, j}+\cdots+\sum T_{i, j}$ intersects the sum of two elements $v, w$ of $V_{i}$ which intersect each other, it follows from (1) and (2) that under its respective metric

$$
\text { diameter } C \cdot(v+w)<1 / 4^{i} \text {. }
$$

If $v$ and $w$ are two elements of $V_{i+1}$ which are subsets of the same element of $V_{i}$, then

$$
E_{i+1}(v, w)<1 / 4^{i+1}+\delta\left(1 / 2^{i+1}+1 / 4^{i}\right)
$$

where $\delta$ is equal to 2,1 , or 0 according as neither, one, or both of the elements $v, w$ are nondegenerate subsets of elements of $K_{i, j}^{\prime}$ for some integer $j$.

Using (3) and (4) we find that if $v$ and $w$ are elements of $V_{i+1}$ which are subsets of the sum of a coherent collection of $n$ elements of $V_{i}$, then

(5) $E_{i+1}(v, w)<n\left(1 / 2^{i}+1 / 4^{i}+1 / 4^{i+1}\right)+1 / 4^{i}<\left(n / 2^{i}\right)\left(1+3 / 2^{i}\right)$.

Using (5) we find that

$$
E_{i+1}(p, q)<\left(1+3 / 2^{i}\right)\left[E_{i}(p, q)+2 / 2^{i}\right] .
$$

Repeated applications of (6) give that

$$
\begin{aligned}
E_{i+k}(p, q)< & \left(1+3 / 2^{i}\right)\left(1+3 / 2^{i+1}\right) \cdots\left(1+3 / 2^{i+k-1}\right)\left[E_{i}(p, q)+2 / 2^{i}\right. \\
& \left.+2 / 2^{i+1}+\cdots+2 / 2^{i+k-1}\right] .
\end{aligned}
$$

Since

$$
\begin{aligned}
\left(1+3 / 2^{i}\right)\left(1+3 / 2^{i+1}\right) \cdots & <\left[1+3 /\left(2^{i}-6\right)\right]\left[1+3 /\left(2^{i+1}-6\right)\right] \cdots \\
& =2^{i} /\left(2^{i}-6\right)
\end{aligned}
$$

then

$$
E_{i}(p, q) \leqq E(p, q)<\left[2^{i} /\left(2^{i}-6\right)\right]\left[E_{i}(p, q)+4 / 2^{i}\right] \quad(i>3) .
$$

That $\lim E_{i}(p, q)=E(p, q)$ exists follows from the fact that $E_{1}(p, q)$, $E_{2}(p, q), \cdots$ is a bounded monotone sequence.

That $E(x, y)$ satisfies the triangle condition follows from the fact that for each triple of points $p, q, r, E_{i}(p, q) \leqq E_{i}(p, r)+E_{i}(r, q)$ $+1 / 2^{i}$. Since $E_{i}(x, y)$ is symmetric, $E(x, y)$ is also; that is, $E(x, y)$ $=E(y, x)$. If $R$ is an open subset of $M$ containing the point $p$, there 
is an integer $n$ so large that $D(p, M-R)>3 / m_{n}$. Since $E_{n}(p, M-R)$ is positive, $E(p, M-R)$ is also. If $p$ and $q$ belong to the closure of the same element of $G_{m_{n+1}}$, then by (4), $E_{n}(p, q)<2 / 2^{n}$, and by $(7), E(p, q)$ $<\left[2^{n} /\left(2^{n}-6\right)\right]\left(2 / 2^{n}+4 / 2^{n}\right)$ if $n>3$. Hence $E\left(p, q_{1}\right), E\left(p, q_{2}\right), \cdots$ has zero as a limit if and only if $q_{1}, q_{2}, \cdots$ converges to $p$. Therefore $E(x, y)$ is a metric for $M$ that preserves its topology.

That $E(x, y)$ is almost convex follows from the fact that for each pair of points $p, q$ and each integer $n$ there is a point $r$ such that $\left|E_{n}(p, r)-E_{n}(p, q) / 2\right|+\left|E_{n}(r, q)-E_{n}(p, q) / 2\right| \leqq 1 / 2^{n}$. Since $M$ is compact, $E(x, y)$ is a convex metric for $M$.

Alternate proof of Theorem 7. The proof of Theorem 7 can be simplified by making use of Theorem 6 of this paper and Theorem 4 of [1] which states that a compact locally connected continuum $M$ has a convex metric if for each point p contained in an open subset $R_{1}$ of $M$, there is an open subset $R_{2}$ of $R_{1}$ containing $p$ such that the boundary of $R_{2}$ with respect to $M$ is a subset of a subcontinuum of $M$ with a convex metric. Hence, we need to show only that $\bar{E}$ of Theorem 6 has a convex metric.

Now $E$ is the sum of a dendron $T_{1}$ and a finite collection $U_{1}$ of connected, mutually exclusive open subsets of $E$ such that the common part of $T_{1}$ and the closure of an element of $U_{1}$ is an end point of $T_{1}$. There is a convex metric $F_{1}$ for $T_{1}$ such that the distance between any two of its end points is between $1 / 2$ and 1 . Let $V_{1}$ be the collection of all continua $v$ such that $v$ is either a point of $T_{1}$ or the closure of an element of $U_{1}$. If $v$ and $w$ are elements of $V_{1}$, define $E_{1}(v, w)$ to be the greatest lower bound of all numbers of the type $r / 2+s$ where some continuum is the sum of $v, w, r$ other elements of $V_{1}$ and arcs in $T_{1}$ whose lengths under $F_{1}$ total $s$.

Let $\epsilon_{2}$ be a number so small that if $v$ and $w$ are two elements of $V_{1}$ which do not intersect, then $D(v, w)>3 \epsilon_{2}$ and $D\left(T_{1}, \bar{E}-E\right)>3 \epsilon_{2}$. Now $E$ is the sum of a dendron $T_{2}$ and a finite collection $U_{2}$ of connected, mutually exclusive open subsets of $E$ such that each element of $U_{2}$ is of diameter less than $\epsilon_{2}$, and the common part of $T_{2}$ and the closure of an element of $U_{2}$ is an end point of $T_{2}$. Let $F_{2}$ be a convex metric for $T_{2}$ that preserves $F_{1}$ on $T_{1}$ and such that any arc in $T_{2}$ is of length between $1 / 2^{2}$ and $1 / 2$ if the end points of this arc are also end points of the closure of a component of $T_{2}-T_{1}$. Denote by $V_{2}$ the collection of all elements $v$ such that $v$ is either a point of $T_{2}$ or the closure of an element of $U_{2}$. If $v$ and $w$ are elements of $V_{2}$, let $E_{2}(v, w)$ be the greatest lower bound of numbers of the type $r / 4+s$, where some continuum is the sum of $v, w, r$ other elements of $V_{2}$ and arcs in $T_{2}$ whose lengths under $F_{2}$ total $s$. 
This process is continued to get a sequence of functions $E_{3}, E_{4}, \cdots$. In general, $\epsilon_{i+1}$ is a number so small that if $v$ and $w$ are any two elements of $V_{i}$ that do not intersect each other, then $D(v, w)>3 \epsilon_{i+1}$ and $D\left(T_{i}, \bar{E}-E\right)>3 \epsilon_{i+1}$. There is a dendron $T_{i+1}$ and a finite collection $U_{i+1}$ of connected, mutually exclusive open subsets of $E$ such that each element of $U_{i+1}$ is of diameter less than $\epsilon_{i+1}$, and the common part of $T_{i+1}$ and the closure of an element of $U_{i+1}$ is an end point of $T_{i+1}$. There is a convex metric $F_{i+1}$ for $T_{i+1}$ that preserves $F_{i}$ on $T_{i}$ such that any arc in $T_{i+1}$ is of length, under $F_{i+1}$, of between $1 / 2^{i+1}$ and $1 / 2^{i}$ if the end points of this arc are end points of the closure of the same component of $T_{i+1}-T_{i}$. Use $V_{i+1}$ to denote the collection of points of $T_{i+1}$ and the closures of elements of $U_{i+1}$. For elements $v$ and $w$ of $V_{i+1}$, define $E_{i+1}(v, w)$ to be the greatest lower bound of $r / 2^{i+1}+s$, where some continuum is the sum of $v, w, r$ other elements of $V_{i+1}$ and arcs in $T_{i+1}$ whose lengths under $F_{i+1}$ total $s$.

Define $E_{i}(p, q)$ to be the greatest lower bound of $E_{i}(v, w)$ where $p$ and $q$ are points of elements $v$ and $w$ respectively of $V_{i}$. The argument to show that $\lim E_{i}(p, q)$ exists and determines a convex metric for $\bar{E}$ is similar to and somewhat easier than that used in the first proof.

Applying Theorem 1 to Theorem 7 we obtain the following.

THEOREM 8. Each compact, locally connected continuum has a convex metric.

The application of Lemma 1 and Theorem 1 to Theorem 7 gives Theorems 9 and 10.

THEOREM 9. Each connected partitionable set has an almost convex metric.

THEOREM 10. Each connected set with property $\mathrm{S}$ has an almost convex metric.

\section{REFERENCES}

1. R. H. Bing, A convex metric for a locally connected continuum, Bull. Amer. Math. Soc. vol. 55 (1949) pp. 812-819.

2. - Extending a metric, Duke Math. J. vol. 14 (1947) pp. 511-519.

3. K. Menger, Untersuchungen über allgemeine Metrik, Math. Ann. vol. 100 (1928) pp. 75-163.

4. E. E. Moise, Grille decomposition and convexification theorems for compact locally connected continua, Bull. Amer. Math. Soc. vol. 55 (1949) pp. 1111-1121.

5. R. L. Moore, Concerning connectedness im kleinen and a related property, Fund. Math. vol. 3 (1922) pp. 232-237. 
6. W. Sierpinski, Sur une condition piur qu'un continu soit une courbe jordanienne, Fund. Math. vol. 1 (1920) pp. 44-60.

7. G. T. Whyburn, Concerning S-regions in locally connected continua, Fund. Math. vol. 20 (1933) pp. 131-139.

8. - Analytic topology, Amer. Math. Soc. Colloquium Publications, vol. 28, New York, 1942.

UNIVERSITY OF WISCONSIN 